\title{
La Salud Mental de los estudiantes
}

\section{universitarios}

\section{Mental Health among University students}

Uno de los muchos temas que hasta hace pocos años no era materia de discusión relevante en círculos docentes y académicos ha sido el de la salud mental de los estudiantes universitarios; las razones eran variadas, pero en todas ellas operaba la noción de que el hecho mismo de haber ingresado luego de aprobar exámenes duros y exigentes constituía una suerte de certificación de un contexto personal, anímico, cognitivo o emocional normal en la mayoría sino en todo el cuerpo estudiantil. Es necesario afirmar ahora que tal no es el caso y que, por el contrario, la convergencia de una serie de circunstancias puede contribuir a que el estudiantado universitario represente más bien una población de riesgo más o menos alto para la ocurrencia de problemas de salud mental (1). El artículo de Sindeev et al. (2), publicado en este número de la Revista Médica Herediana, así lo demuestra.

El ingreso a una institución de educación superior tiene lugar en una fase decisiva del ciclo vital de todo ser humano: el final de la adolescencia y el comienzo de la adultez joven. No se trata solamente de la materialización inicial de aspiraciones orientadas a un largo futuro, a una carrera que engrana vocación profunda y anhelo intenso de satisfacciones y éxito. Este proceso tiene lugar en un momento en que el/la estudiante afronta también cambios psicofisiológicos marcados, afrontes socio-existenciales complejos y hasta impredecibles, un entorno a veces radicalmente diferente generado por alejamientos familiares que no por planeados dejan de ser experiencias nuevas y desafíos formidables (3).

Esta etapa, la de la vida universitaria entraña, particularmente en los primeros años, la experiencia de activos factores de riesgo y el eventual uso de factores de protección, todos ellos actuando en el escenario de predisposiciones personales que los clínicos llaman personalidad o identidad, un conglomerado de variables bio-psico-socio-culturalespirituales que incitan o atenúan éxito o fracaso en la nueva etapa vital (4). Entre los factores de riesgo, se cuentan la renovada complejidad del área de estudios escogida, el reto cuotidiano a la autenticidad y la solidez de la vocación que abrió la ruta del estudiante a sus nuevos claustros, la calidad íntima de una adaptabilidad no expuesta a pruebas similares en el pasado, la competitividad con los nuevos amigos o adversarios, la diferente urdimbre de bullying, conflictos, romances o - tal vez peor aún - indiferencia de los flamantes condiscípulos. El desenlace de estas circunstancias puede tomar muchos años.

El/la estudiante universitario(a) utilizará también los llamados factores de protección, una gama de recursos que cumplen roles de defensa, de afrontamiento, de manejo individual o colectivo de situaciones estresantes, de fomento y forja de fuentes de esperanza, tenacidad, empatía y coraje. Más allá de los componentes individuales o personales, entran en vigencia la cautivante atracción de los temas o actividades académicas que condujeron al alumno a esta nueva ruta de su formación, las satisfacciones iniciales de un conocimiento inédito, el apoyo profundo y leal de la familia cercana o lejana, las nuevas amistades forjadas en el altar de intereses académicos comunes.

No obstante, todo ello puede no ser suficiente para refrenar vivencias anómalas que van desde rasgos o síntomas más o menos aislados hasta manifestaciones psicopatológicas en urgente necesidad de tratamiento primario o especializado. Se incluyen en esta área clínica cuadros de depresión, ansiedad, abuso de sustancias, acentuación de rasgos de personalidad anormal y varios otros. En los grupos etarios correspondientes al estudiantado universitario, los síntomas tanto cognitivos como afectivos son interiorizados y se presentan con una mayor intensidad. Son relativamente frecuentes también los trastornos conductuales, conducta sexual promiscua, agresividad, retraimiento, humor fluctuante (5). 
En este contexto, es conocida la prominencia de depresión y sus variantes nosológicas en la llamada Carga Global de Enfermedad (6); dentro de ella, se da una variedad de las llamadas conductas suicidas: desde pensamientos de muerte hasta consumación del acto, pasando por franca ideación suicida y una variedad de gestos o actos autodestructivos de reiterada frecuencia e intensidad creciente (7). Al igual que en otros segmentos poblacionales en nuestro país y en el mundo, ésta es una realidad hoy innegable entre muchos miembros de comunidades estudiantiles universitarias.

Resulta pues urgente que las instituciones académicas actúen en varios frentes a fin de controlar o aliviar esta situación que parece afectar a, por lo menos, una cuarta parte de su población estudiantil. En términos preventivos, se trata de brindar a todo nivel un ambiente acogedor, positivo, ameno, claramente orientado al bienestar anímico, espiritual e intelectual de los estudiantes. Desde la planta física hasta la actitud de los docentes, desde las tareas de enseñanza y aprendizaje hasta las actividades culturales o sociales, la institución universitaria debe hacer honor a su finalidad fundamental: formar profesionales competentes que posean, al mismo tiempo, auténtica calidad humana, plena estabilidad emocional y una visión integral de su futuro. Pueden contribuir a ello, medidas específicas aun antes del proceso formal de admisión y matrícula que debe ser, por definición, objetivo y completo; en otras palabras, una de las responsabilidades definidas de la universidad, desde el primer momento en que asume sus tareas pedagógicas, es velar continua y plenamente por el bienestar de todos sus estudiantes.

Esta tarea va más allá, sin embargo. La universidad debe forjar una cultura abierta y objetiva de mantenimiento de ese bienestar y de oportunidades de búsqueda de ayuda profesional especializada si el estudiante enfrenta momentos o experiencias difíciles. La existencia de centros de atención médica específicamente orientados al resguardo de la salud integral del alumnado es condición indispensable y que el campo de la salud mental cuente con personal y ambientes apropiados de evaluación es, obviamente, un aspecto fundamental de estos programas. La disponibilidad de los docentes como asesores o consejeros constituye un paso saludable de confianza, diálogo y, eventualmente, detección temprana de problemas que requieran referencia a aquellos Centros. La privacidad y el pleno apoyo institucional (incluyendo comunicación con familiares, cuando fuere necesario) deben ser garantizados.

La evaluación clínica puede conducir a decisiones o medidas terapéuticas de magnitud diversa, dictadas por la naturaleza del diagnóstico al que se arribe. Pueden incluir consejería mental, psicoterapia, farmacoterapia y hasta referencias de hospitalización en caso necesario. La universidad debe alentar decididamente al estudiante afectado a implementar tales sugerencias, contando deseablemente con un claro apoyo familiar. En todo caso, la responsabilidad institucional abarca el seguimiento que, desde la perspectiva académica, tiene que ver con los pasos subsecuentes en la carrera del (o la) docente.

En suma, la salud mental del estudiantado universitario es un aspecto fundamental en la conducción de la educación superior, garantía esencial de una trayectoria exitosa. Hoy en día, al prestársele más atención que hace algunas décadas, se detecta una mayor prevalencia de una variedad de cuadros clínicos de severidad diversa, dentro de los cuales predominan estados de depresión y ansiedad (8). La responsabilidad institucional se extiende a tareas de prevención, constante apoyo ambiental, disponibilidad de oficinas y personal a cargo de labores de diagnóstico temprano, variado manejo inicial y pertinentes disposiciones adicionales, dictadas por una evaluación integral del caso individual. El impacto positivo de medidas de esta naturaleza ha de ser evidente.

Renato D. Alarcón 1,a

\section{REFERENCIAS BIBLIOGRÁFICAS}

1. Shackle S. "The way universities are run is making us ill": Inside the student mental health crisis. New York: The Guardian; 27 de setiembre del 2019.

2. Sindeev A, Arispe CM, Villegas JN. Factores asociados al riesgo e intento suicida en estudiantes de medicina de una universidad privada de Lima. Rev Med Hered. 2019; 30:232-241.

3. Wiener J, Dulcan M. Tratado de Psiquiatría de la Infancia y la Adolescencia. Barcelona, España: Masson; 2006.

4. Eisenberg D, Hunt J, Speer N, Zivin K. Mental Health service utilization among College students in the 
United States. J Nerv Ment Dis. 2011; 199(5):301308.

5. Fernández A. Trastornos psiquiátricos durante la infancia y la adolescencia. En: Introducción a la Psiquiatría (Suárez Richards M.,Comp), 4a Ed. Buenos Aires, Argentina: Editorial Polemos; 2012. pp. 487-522.

6. Lopez AD, Murray CJL. The global burden of disease, 1990-2020. Nature Med. 1998; 4:1241-1243.
7. Mann JJ, Bortinger J, Oquendo MA, Currier D, Shuhua L, Brent DA. Family history of suicidal behavior and mood disorders in probands with mood disorders. Am J Psychiatry. 2005; 162(9):1672-1679.

8. Givens J, Tja J. Depressed medical students' use of mental health services and barriers to use. Acad Medicine. 2002; 77(9):918-921.

1. Profesor Emérito. Facultad de Medicina Alberto Hurtado, Universidad Peruana Cayetano Heredia, Lima, Perú.

a. Titular de la Cátedra Honorio Delgado 